\title{
Revisiting Characters of Human Immune Orchestra in Light of Immuno-Suppression by Corona Virus
}

\section{SS Jha*}

Director, Mahavir Vaatsalya Aspatal, Patron and Founder President, Indian Orthopaedic Rheumatology Association, Vice Secretary General, Asia-Pacific Society for Foot and Ankle Surgery, Past President, Indian Foot and Ankle Society, Patna, Bihar, India

*Corresponding Author: SS Jha, Director, Mahavir Vaatsalya Aspatal, Patron and Founder President, Indian Orthopaedic Rheumatology Association, Vice Secretary General, Asia-Pacific Society for Foot and Ankle Surgery, Past President, Indian Foot and Ankle Society, Patna, Bihar, India

Basic understanding of immunology is relevant today in the light of consciousness raised by the worldwide pandemic of SARSCOV-2 responsible for Novel COVID-19.

Immune system is evolved to protect from universe of foreign pathogens. During the course of evolution, depending on species, there has been diversification and high sophistication to permit the organism to adapt to its environment. The human immunity has two interdependent arms, "innate" and "adaptive" immune systems.

The cellular and molecular components of these immune systems interact to provide robust host defence mechanisms through cellular and humoral immunity. The aberrations of immunity predispose to chronic inflammatory immune mediated pathology.

"Innate" immune responses are immediate and in rapid succession eliminating the 'invaders' by phagocytosis. Activation of this innate system follows recognition of molecular pattern expressed by the invader prokaryotic primitive invertebrate organism like bacteria. Molecular patterns are not expressed by eukaryotic cells present in the host tissue.

Lipopolysaccharide present in bacteria and double stranded RNA present in retroviruses are recognised by receptors of the host like in invertebrates focussing the innate system only.

This activation of the innate immune system by recognition of microbial constituents is an engenius and simple mechanism to discriminate between self and non-self, which initiates rapid and strong response to the invading micro-organism.

Priming of activation of innate immune cells

On initial encounter with microorganisms, activation of innate immune cells gets primed. This priming during subsequent encounters gets deliberated non-specific and limited. Importantly, a second- encounter by the same microbe will not result in a response in a more specific way.

Initial 800 million years of evolution, another arm of immune system evolved during subsequent 480 million years as "adaptive system" along with interdependent innate immunity in higher species starting from fish, amphibians, reptiles, birds and mammals etc.

The adaptive immune system is relatively slow in regards to the invader on first encounter. Specific cells of the adaptive system are the $\mathrm{T}$ cells. They take time to get activated in the draining lymph nodes, where these cells first divide and then only become functional.

Compared to the innate system, the adaptive system recognize pathogens in highly specific fashion. They have the ability to form much stronger immunological memory. During repeat infection, they respond rapidly like innate system.

The resultant memory $\mathrm{T}$ and $\mathrm{B}$ cells from the basis of vaccination success like in small fox.

The immune system achieves an equilibrium between favouring host defence against foreign pathogens and protecting host tissues from collateral damage.

Tilt in this delicate balance becomes pathological. In immune deficiency, emergence of serious infection or occasionally a neoplastic disease can be promoted. Conversely, with sustained and unbridled immune response or badly adapted immune system can trigger allergic, inflammatory and autoimmune disease.

\section{Cellular players in immune symphony}

The immune symphony is performed in the human body consisting of

1. Circulatory Pathway

2. Lymphatic Pathway

3. Special dressing rooms

- Lymphatic organs

4. Sanctuary areas-1

- $\quad$ Eye

- Testicle

5. Dedicated organs-specific

- Shin 
- Joints

- Kidney

Symphony conductors are central lymphoid organs:

- Thymus

- $\quad$ Bone marrow, where the cell (T \& B Cells), the immune get to train themselves.

Secondary central lymphoid organs-spleen lymph nodes and mucus membrane are seats of remedial courses.

The musicians of the innate immune system are cells like - polymorphs, leucocytes, monocytes/macrophages, dendritic cells, and natural killer cells (NK).

The musicians of the adaptive immune system are T and B cells, they require to be trained.

CD - Various surface molecules differentiate the cells of the immune system from each other. These molecules are termed - cluster of differentiation. Each immune cell has specific CD molecules.

The 'instruments' are substances synthesized to establish communication between cells. High affinity receptors receives the communication and become capable of interacting with specific ligands such as soluble molecules - cytokines and chemokines exercising different functions.

\section{Bone marrow and lymph node/spleen}

Progenitor generating centre of the immune system is the bone marrow. Most immune cells originate here and migrate to the peripheral blood, lymphoid organs and thymus. Pluripotent precursor cells in the bone marrow produce myeloid progenitor cells and subsequently polymorphonuclears monocytes, macrophages and dendritic cells.

The lymphoid progenitor cells in bone marrow produce $\mathrm{T}$ and $\mathrm{B}$ cells and a specific cell the N K Cells.

Lymph node/spleen/peripheral lymphoid organs

These are places for lodgement of dendritic cells already loaded with captured antigens. T and B cell activation is facilitated.

Role of lymphoid tissues with mucus membrane

Mucus membrane is crucial for host defence as they are spread to constitute a massive surface area of tissue in lungs and intestine and direct contact with the environment.

The lymphocyte infiltrate lodged. There is called -MALT-Mucosa-associated lymphoid tissue either as bronchial or gut associated. Tonsil and appendix are such enriched areas too.

This MALT system functions like the lymph nodes and spleen allowing special interactions between Antigen Presenting Cells (dendritic cells) and lymphocytes. This MALT system has a unique characteristic that it may appear and disappear depending on the environmental stimuli.
It is now accepted that this lymphoid neogenesis is seen in synovium and its persistence is seen in rheumatoid arthritis and other chronic immune pathological conditions.

Immune regulation and generation of specific effector defence responses are coordinated in the lymphoid organs. Cell contact dependant pathways and though less well defend also exist.

Immune regulation is based on interaction, activation and expansion of specific subsets of T and B cells.

\section{Aberrations of immunity}

Dysregulation of immune response results into the pathogenicity of multiple immune-mediated inflammatory diseases. The two extremes of this dysregulation are having either deficiency or with overactive (inappropriately persistent) immune responses.

\section{Immune deficiencies}

Inherited immune deficiencies are $\mathrm{x}$-linked, monogenic and recessive. Each one is specific clinical disease entity guided by genetic anomaly and interplay of cellular or molecular immunity.

\section{Acquired immune deficiencies}

Acquired immune deficiencies, AIDS caused by a virus which destroys $\mathrm{T}$ cells, dendritic cells and macrophages. It gains access through high affinity interactions with cells surface receptors like CD4 co-receptor and chemokine receptors. Use of glucocorticoid, cell depleting biological treatment further augment the acquired immune deficiencies.

\section{Auto-inflammatory syndromes}

These are inherited diseases with persistent, systemic and have chronic inflammatory responses. They are monogenic arising through poorly understood aberrations of inflammatory signalling pathways. Two groups of such diseases are:

1. Certain forms of Crohn's disease and other related granulomatous diseases. Excess production of uric acid with precipitation to form urate crystals also might stimulates similar intracellular pathways leading to inflammatory syndrome-GOUT. Adult stilts disease or PFAPA syndrome (Periodic fever, Aphthous stomatitis, Pharyngitis, Adenitis)-mechanism remains unknown but may turn out to be inherited polygenic disorder.

2. Recurrent hereditary fevers comprising familiar Mediterranean fever, cold urticaria and CINCA (chronic infantile neurological cutaneous and articular syndrome - these diseases are considered to be inherited disturbances of the innate immune response.

\section{Auto-immune diseases}

Rheumatoid arthritis/Type I diabetes are most probably caused by response of the cells of the adaptive immune system i.e. T and B cells to tissues of the host. Autoantibodies are found which is specific for the particular disease invariably HLA system has an associ- 
ation indicating the involvement of $\mathrm{T}$ cells. Cure of an autoimmune disease is often difficult because

\section{B and T cells forms memory cells}

2. Autoimmune response is directed against an antigen expressed by a person's own body. Exact aetiology of autoimmune diseases evade and answer but they involve multiple mechanisms.

\section{Lymphoproliferative disease}

Deregulation of the basic mechanisms, such as apoptosis as well as chronic stimulation arising through persistent infection i.e. Epstein-Barr virus infection:

1. Sjogren's Syndrome - this autoimmune disease with increased incidence of D cell lymphoma is considered to be because of chronic immune stimulation linked to persistent antigenic stimulation.

2. ALPS and follicular lymphoma-the "malignant" lymphocytes proliferate since the immune system no longer eliminates.

\section{SARS-CoV-2}

SARS-CoV-2, which caused the 2019-20 pandemic of coronavirus disease 2019. Severe acute respiratory syndrome coronavirus 2 (SARS-CoV-2), previously known by the provisional name 2019 novel coronavirus (2019-nCoV) is a positive-sense single-stranded RNA virus.

SARS-CoV-2 has close genetic similarity to bat coronaviruses, suggesting it emerged from a bat-borne virus. An intermediate animal reservoir such as a pangolin is also thought to be involved in its introduction to humans.

The basic reproduction number of the virus has been estimated to be between 1.4 and 3.9. The reproduction number may be higher in densely populated conditions such as those found on cruise ships and aircrafts.

Human-to-human transmission of the virus has been confirmed in all of these regions.

This enveloped single stranded RNA virus enters the host cell by binding to ACE2 (human angiotensin converting enzyme II) receptor.

The virus uses human ACE2 molecule as its entry receptor in lungs- this is considered a HALLMARK OF TRANSMISSIBILITY, the receptor binding domain is located in the amino-terminal regions, amino acids of the SARS-COVID protein - directly involved in binding to ACE2. It has ability to use human ACE2.

ACE has a role in both innate and adaptive immune responses by modulating macrophage and neutrophil function. These effects are further magnified when these cells over expressed ACE. ACE is a zinc - dependant dicarboxypeptidase. It originally plays a major part in blood pressure regulation by converting angiotensin I to angiotensin II.

Neutrophils over expressing ACE have an increased production of superoxide increasing their ability to kill bacteria. Expression levels of ACE are particularly high in lungs, kidney and placenta etc.

ACE is primarily located in cell membrane.

Macrophages have a crucial role as antigen presenting cells by interacting with $\mathrm{T}$ cells in adaptive response.

\section{Effect of ACE inhibitors on immune response}

Extensive evidence in experimental animals indicates that inhibition of ACE typically suppresses to autoimmune process. In humans, little information on its effectiveness in treating rheumatoid arthritis, though one small cohort study suggest a positive effect of ACE inhibition in $66 \%$ patients. Additional human study can only lead to better mechanistic understanding of how exactly ACE effects the immune response. This is a key area of interest and holds great promise for novel therapies.

\section{Assets from publication with us}

- Prompt Acknowledgement after receiving the article

- Thorough Double blinded peer review

- Rapid Publication

- Issue of Publication Certificate

- High visibility of your Published work

Website: https://www.actascientific.com/

Submit Article: https://www.actascientific.com/submission.php Email us: editor@actascientific.com

Contact us: +919182824667 